\title{
An Experimental Examination of the Volunteer's Dilemma
}

\author{
Jacob K. Goeree, Charles A. Holt, Angela M. Smith*
}

\begin{abstract}
In a volunteer's dilemma, only one "volunteer" is needed to obtain a benefit for all. Volunteering is costly, and the symmetric Nash equilibrium involves randomization. These predictions have the intuitive property that volunteer rates decline with larger groups, but surprisingly, the probability of obtaining no volunteers is increasing with group size, even as the number of players goes to infinity. These predictions are evaluated in a laboratory experiment with a range of group sizes. Observed volunteer rates are lower with larger groups, as predicted, but the incidence of no-volunteer outcomes declines with group size, in contrast to theory. This reduction in no-volunteer outcomes for large groups can be explained by a one-parameter generalization of the Nash equilibrium that adds quantal response "noise" due to unobserved random effects. Significant individual heterogeneity in observed volunteer rates motivates the estimation of a heterogeneous equilibrium model with a distribution of propensities for volunteering.
\end{abstract}

JEL Codes: C57, C72, C92

\section{Introduction}

A classic paradigm in public economics is the voluntary contributions game in which each person in a group must decide whether to make a costly contribution that provides a benefit to all group members, or to "free ride" on others' contributions. In a "provisionpoint" version of this game, the public benefit is not obtained unless a pre-specified level of contributions is reached. There is a special category of voluntary contributions games where the provision point is that only a single contributor or "volunteer" is needed for the pubic good to be obtained. For example, it takes just one person to veto an undesired outcome under unanimity voting, but such vetoes may be politically costly. Similarly, all members of a legislative body may desire a pay raise, but each prefers that someone else

\footnotetext{
* Goeree: University of Technology, Sydney Australia NSW 2007. Holt: Economics, P.O Box 400182, University of Virginia, Charlottesville, VA 22904-00182. Smith: Economics, James Madison University, MSC 0204, 421 Bluestone Drive, Harrisonburg, VA 22807. This research is funded in part by the National Science Foundation (SBR 0094800 and 1459918) and the University of Virginia Bankard Fund. We thank Megan Porzio, Daniela Puzzello, Emily Snow, and Michelle Song for suggestions and comments.
} 
incurs the cost of sponsoring the bill. Another example can be found in the behavior of a group of foraging animals in which one of them will occasionally look up and check for a predator. The one who issues an alarm is more likely to attract the attention of the predator but helps ensure the safety of others. It has been observed that ground squirrels check more frequently in the presence of kin (Murnighan, Kim, and Metzger, 1993).

The game where only one contribution is needed to provide the group benefit is known as the "volunteer's dilemma" (Diekmann 1985, 1986). Specifically, let $V$ denote the monetary payoff that all $N$ members of a group receive if at least one of them decides to incur a cost $C$. If nobody volunteers, then all receive a lower payoff of $L<V$. It is assumed that $C<V-L$, so each person would prefer to volunteer if nobody else does. But if someone else is expected to volunteer, then each of the others would prefer to "free ride." Thus there are many asymmetric equilibria in which one person volunteers and the others do not, but coordinating on such equilibria may be difficult when decisions must be made quickly or simultaneously, as when bystanders must decide whether to rush in and attempt a risky rescue of someone in trouble.

In the volunteer's dilemma with payoffs that satisfy the assumptions made above, there is also a symmetric mixed-strategy Nash equilibrium in which each person volunteers with probability $p$. Not surprisingly, the equilibrium level of $p$ is a decreasing function of the group size, $N$. This prediction is consistent with casual observation and is also seen in results from social psychology experiments with a staged "emergency" (Darley and Latane, 1968; Latane and Darley, 1970). This diminished tendency for members of large groups to intervene in staged emergencies has been attributed to a "diffusion of responsibility." Public awareness of this issue was heightened by the 1964 fatal stabbing of Kitty Genovese in the courtyard of her apartment complex in full view of a large number of neighbors (although the accuracy of some newspaper accounts has later been disputed). A less tragic outcome occurred when Teresa Saldana, who appeared in the 1982 film Raging Bull, was attacked by a crazed fan who had come to Los Angeles from Scotland to stalk her. Her screams attracted a group of bystanders, but only one man making a bottled water delivery charged in and risked injury or death to hold the assailant down until police arrived. The actress survived to continue with her career. 
Even though the individual probability of volunteering declines with group size, it may be the case that the probability of getting at least one volunteer does not decline as the number of potential volunteers increases. This possibility may seem intuitive, but it is at odds with the symmetric Nash equilibrium. For example, if $V=\$ 1.00, C=\$ 0.20$, and $L=\$ 0.20$, it will be shown in the next section that the Nash prediction is for individual volunteer rates to decline from $3 / 4$ to $1 / 2$ as the group size increases from 2 to 3 . So the chances of getting at least one volunteer goes from $1-(1-(3 / 4))^{2}=0.94$ for $N=2$ down to $1-(1-(1 / 2))^{3}=0.88$ for $N=3$. Moreover, as $N$ goes to infinity, the Nash prediction for the probability of getting at least one volunteer falls to 0.75 . Franzen (1995) tested this unintuitive prediction for group sizes ranging from 2 to 101 . The volunteer rate fell from about $2 / 3$ for small groups to about half that rate for large groups, and the probability of getting at least one volunteer was close to 1 for groups with 10 or more people. Subjects in this experiment completed a questionnaire and received results and monetary payments later by mail.

There can be considerable "noise" and confusion in a one-shot game, due to calculation errors and unobserved preference shocks, which may persist in repeated games with random matching. As a consequence, the probability of getting at least one volunteer could approache 1 for large groups. McKelvey and Palfrey (1995) proposed a generalization of the Nash equilibrium, the quantal-response equilibrium (QRE), which incorporates noise effects in an equilibrium framework that requires choice probabilities to be consistent with beliefs. The symmetric QRE prediction is that the probability of getting at least one volunteer will approach 1 for sufficiently large groups (Goeree and Holt, 2005). Equilibrium theories should be tested under conditions in which beliefs have had a chance to stabilize, and therefore this paper will report the results of repeated volunteer's dilemma games with random matching. ${ }^{1}$ Note that it would be difficult for subjects to somehow coordinate on asymmetric equilibria with random matching, and therefore, our analysis will focus on the symmetric equilibrium predictions. In the online

\footnotetext{
${ }^{1}$ Many volunteers' dilemma situations (e.g. saving a victim) are not repeated. Random matching is a standard method of providing subjects with a series of 1-shot interactions in which they are able to learn about others' behavior in a general sense, without learning about the prior behavior of the specific people with which they are currently matched.
} 
appendix, however, we do provide a detailed comparison of volunteer rate and variance predictions for symmetric and all possible asymmetric equilibria.

While theoretical explorations into the volunteer's dilemma are widespread, experimental evidence is relatively limited. Healy and Pate (2009) consider the effects of announced cost asymmetries, while others (i.e. Otsubo and Rapoport, 2008) examine a more dynamic volunteer's dilemma game with a finite horizon and a decreasing benefit of the public good over time. Otsubo and Rapoport find that subjects tend to volunteer earlier than predicted by theory, have difficulty coordinating on asymmetric equilibria, and volunteer more when the cost is lower. They also observe a large degree of heterogeneity across subjects in their free-riding behavior, a result that is featured in our data as well. ${ }^{2}$ The decision of whether to volunteer or not depends on beliefs about others' decisions. Babcock, Recalde, Vesterlund, and Weingart (2016) consider the effects of gender sorting and find evidence that people believe that women are more likely to volunteer in mixed-gender groups. Finally, Bergstrom, Garratt, and Leo (2015) consider an interesting variation in which one person, designated as the victim, receives a benefit if at least one other volunteers. They elicited utility payoffs from participants, using a timed mechanism to select a contributor. Subjects are motivated in part by interpersonal comparisons of volunteer decisions in this game.

A final issue to be addressed is the nature of any biases that might cause volunteer rates to deviate systematically from Nash predictions. Both Diekmann (1986) and

\footnotetext{
${ }^{2}$ Diekmann (1985) notes another unintuitive feature of the mixed-strategy Nash equilibrium in asymmetric volunteer's dilemma games. Since each person must be indifferent between their two decisions in order to be willing to randomize, the expected payoffs for volunteering and not volunteering must be equal. In a two-person game, an increase in one person's volunteer cost will not alter that person's predicted volunteer rate, since any change would cause the other person to no longer be indifferent. But the person whose cost has increased must be "induced" back to indifference by lowering the equilibrium volunteer rate of the person whose cost has not changed. Recall the numerical example given above $(V=\$ 1.00, C=\$ 0.20, L=$ $\$ 0.20$ ), which produces a Nash volunteer rate of 0.75 with $N=2$. It can be shown that a three-fold increase in one person's cost, from $\$ 0.20$ to $\$ 0.60$, will not alter that person's equilibrium volunteer rate but will reduce the other person's rate from 0.75 to 0.25 . This absence of an "own-payoff effect" is unintuitive, and such effects have been observed and explained by a quantal-response approach in some other asymmetric 2x2 games with unique mixed-strategy equilibria (Goeree, Holt, and Palfrey, 2003). Diekmann (1985) provides some evidence for own-payoff effects in 2-person volunteer's dilemma games, although the results for 5-person games were more ambiguous. As was the case with the Franzen (1995) experiment discussed above, these asymmetric-payoff experiments were done as one-shot games in which subjects filled out questionnaires and received results and payoffs by mail. More recently, Healy and Pate (2009) find experimental evidence that individual volunteer rates decrease both with an increase in own cost and a decrease in other group members' costs.
} 
Franzen (1995), for example, report volunteer rates that were generally in excess of Nash predictions for all group sizes in one-shot games administered via a questionnaire. This tendency for excess cooperation might be explained by altruism, confusion, or misaligned expectations in games played only once. In the laboratory experiments with repeated random matchings that are reported in this paper, volunteer rates are too low for small group sizes (2 and 3), and are slightly above Nash predictions for larger group sizes (6, 9, and 12). The volunteer's dilemma is an ideal setting in which to consider behavioral noise, since the outcome can be sensitive to the decision of a single individual. ${ }^{3}$ Once randomness in decision-making is included, there seems to be a downward bias in volunteer rates for all treatments. This downward bias could be due to the fact that individuals have a "cool" glow propensity to free-ride by not volunteering. Alternatively participants may anticipate some regret associated with a volunteer decision when it turns out to be unneeded. Therefore, we will consider a stochastic (quantal response) model with individual-specific propensities or aversions to volunteering that could be due to some of these potential biases. It is reasonable to expect that individuals would differ in terms of sensitivity to behavioral biases, and this conjecture is consistent with the observed variation in individual volunteer rates. The heterogeneous quantal response model allows for these differences by permitting a distribution of individual "types" (propensities or aversions, depending on the specification). ${ }^{4}$ The equilibrium requires that beliefs about others' volunteer probabilities match the proportions of volunteer decisions that are jointly determined by these beliefs, by the distributions of individual types, and by any residual "noise" in the decision-making process. The estimation process is innovative in that the equilibrium (in which beliefs match decision proportions) must be solved for at each stage of the iterative search for parameters that maximize the likelihood function.

\section{The Symmetric Nash Equilibrium}

A decision to volunteer results in a sure payoff of $V-C$, and a decision not to volunteer yields the high payoff, $V$, if at least one other person volunteers. In a

\footnotetext{
${ }^{3}$ Other games with this type of sensitivity include jury voting under a unanimity rule, or coordination games where the lowest effort determines all players' payoffs.

${ }^{4}$ See Goeree, Holt, and Palfrey (2016, Chapter 5) for a general specification of a heterogeneous quantal response equilibrium and relevant references.
} 
symmetric equilibrium, all players volunteer at a rate $p$, so the probability of getting at least one volunteer decision from the $N-1$ others is: $1-(1-p)^{N-1}$. Recall that the payoff if nobody volunteers is $L$, so the expected payoff from not volunteering is provided on the right side of (1). In order to be willing to randomize, this expected payoff must equal the sure volunteer payoff of $V-C$ on the left side of (1):

$$
V-C=V\left[1-(1-p)^{N-1}\right]+L(1-p)^{N-1} .
$$

Thus the symmetric Nash equilibrium probability of not volunteering is:

$$
1-p=\left(\frac{C}{V-L}\right)^{\frac{1}{N-1}}
$$

and hence the equilibrium volunteer rate is determined:

$$
p=1-\left(\frac{C}{V-L}\right)^{\frac{1}{N-1}} .
$$

Therefore the volunteer rate will be decreasing in $N$ if $C /(V-L)<1$, as assumed.

Next consider the probability that none of the $N$ players decide to volunteer, which is $(1-\mathrm{p})^{N}$. This probability can be calculated from (2):

$$
\operatorname{Pr}(\text { no-volunteer outcome })=(1-p)^{N}=\left(\frac{C}{V-L}\right)^{\frac{N}{N-1}},
$$

which is increasing in $N$ when $C<V-L$, as assumed.

\section{Procedures}

Cohorts of subjects participated in 20 consecutive decision periods, with random matching, although the number of periods was not announced. The game is symmetric in that the benefit to every member of the group was $\$ 1.00(V)$ if at least one person volunteered, and the individual cost of volunteering was $\$ 0.20(C)$ for each person. In the absence of any volunteers, each person in the group earned $\$ 0.20(L)$. Group size $(N)$ varied across treatments and took one of five values: $2,3,6,9$, or 12 . These group sizes were chosen because they induce Nash equilibria on both sides of $1 / 2$. Specifically, as can be seen from equation (3), the symmetric Nash equilibrium volunteer probability falls from 0.75 to 0.5 to about 0.25 as the group size increases from 2 to 3 to 6 . Three sessions were conducted in each of these three treatments, two at the University of Virginia and 
one at UCLA. In addition, we used the large lab at UCLA to run a session with 24 participants (4 groups of size 6), a session with 36 participants (4 groups of size 9), and a session with 48 participants (4 groups of size 12). The two "out-of-sample" test sessions with group size 3, described in section VII, were conducted at the University of Virginia.

Participants were seated at visually isolated computer terminals. Instructions were both displayed on the subjects' screens and read aloud by an experimenter. Neutral terminology was used to avoid any bias due to the volunteer's dilemma scenario. Thus, each individual chose whether to "invest" or "not invest," where investment by any member of the group at a cost would provide a monetary benefit to all members of the group, regardless of whether they chose to invest. ${ }^{5}$ After each round, subjects were told the number of volunteer decisions (if any) for their group in that round. Subjects were undergraduate students recruited from classes at the University of Virginia and UCLA. Each subject was paid $\$ 6$ for showing up and any additional earnings from the experiment. Total earnings for the experiment, which lasted approximately 45 minutes, averaged about $\$ 21$ and were paid in cash at the conclusion of the experiment.

\section{Data}

The results for all sessions are reported in the tables below. Table 1 displays average volunteer rates by session, along with treatment averages and Nash predictions. The data for the UCLA sessions are shown in italic type, and it is apparent that there is no obvious subject pool difference. The session averages are ranked inversely with group size, as predicted.

Table 1. Volunteer Rates (UCLA Sessions in Italics)

\begin{tabular}{cccccc}
\hline \hline & $\begin{array}{c}N=2 \\
(34 \text { subjects })\end{array}$ & $\begin{array}{c}N=3 \\
\text { (36 subjects) }\end{array}$ & $\begin{array}{c}N=6 \\
(48 \text { subjects })\end{array}$ & $\begin{array}{c}N=9 \\
(36 \text { subjects })\end{array}$ & $\begin{array}{c}N=12 \\
(48 \text { subjects })\end{array}$ \\
\hline Session Averages & $0.55,0.51,0.49$ & $0.42,0.38,0.39$ & $0.28,0.20,0.31$ & 0.19 & 0.19 \\
Overall Average & 0.52 & 0.40 & 0.28 & 0.19 & 0.19 \\
Symmetric Nash & 0.75 & 0.50 & 0.24 & 0.16 & 0.12 \\
\hline
\end{tabular}

\footnotetext{
${ }^{5}$ The instructions are provided in the Appendix. The software interface used to conduct the experiment can be found on the Veconlab website: (http://veconlab.econ.virginia.edu/vd/vd.php).
} 
The averages by treatment, along with the symmetric Nash predictions, are also plotted in Figure 1. The small dotted gray lines around the Nash predictions show 95\% confidence intervals around the predicted symmetric Nash equilibrium volunteer probability. ${ }^{6}$ The data averages are declining in group size, but they do not reflect the predicted degree of sensitivity to this variable.

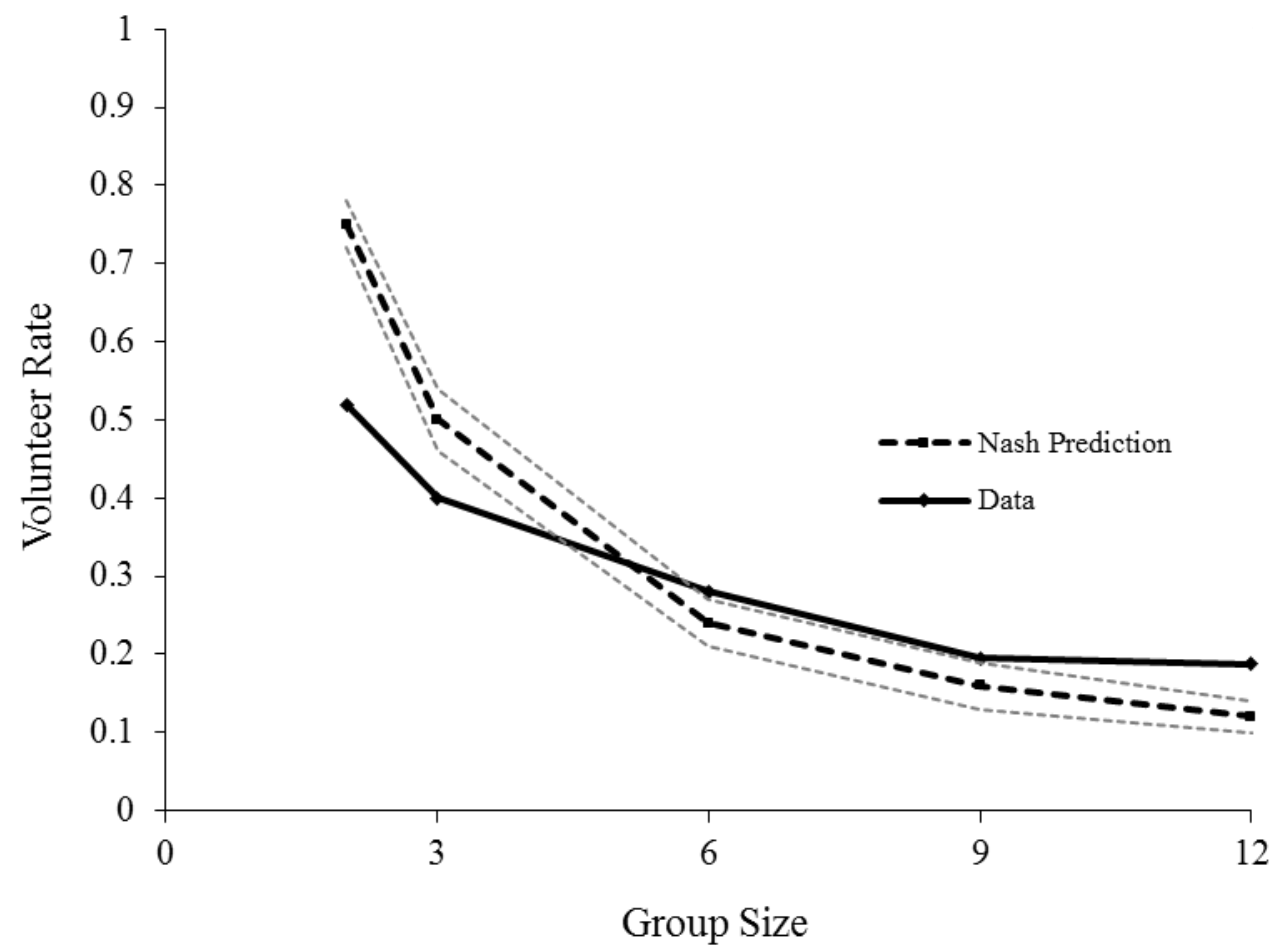

Figure 1. Volunteer Probabilities: Nash predictions (thick dashed line) with corresponding $95 \%$ confidence interval bounds (thin dashed lines) and observed average (solid line)

Instead of ranging from 0.75 to 0.12 , as predicted in a symmetric Nash equilibrium, the observed average volunteer rates are much more condensed and range only from 0.52 to 0.19. There seems to be some underlying influence that causes observed probabilities to

\footnotetext{
${ }^{6}$ Given the Nash equilibrium predicted volunteer probability and the number of trials in the data for each treatment $(680,720,960,720$, and 960 for $N=2,3,6,9$, and 12, respectively), we can determine the cumulative distribution function for the number of volunteer decisions using the binomial density function. The lower bound represents the volunteer rate corresponding to the number of trials where the cumulative distribution is 0.025 and the upper bound represents the volunteer rate corresponding to the number of trials where the cumulative distribution is 0.975 .
} 
be lower relative to the Nash prediction for smaller group sizes and higher for larger group sizes. Some explanations for this pattern will be considered in the next section.

As noted above, one unintuitive Nash prediction is that the probability that no members of a group volunteer actually increases with group size. The incidence of novolunteer outcomes is reported in Table 2 for each session, along with Nash predictions and treatment averages, which are also plotted in Figure 2. The small dotted gray lines around the Nash predictions show 95\% confidence intervals around the predicted rate of no-volunteer outcomes. ${ }^{7}$

Table 2. Rate of No-Volunteer Outcomes (UCLA Sessions in Italics)

\begin{tabular}{cccccc}
\hline \hline & $\begin{array}{c}N=2 \\
(34 \text { subjects })\end{array}$ & $\begin{array}{c}N=3 \\
\text { (36 subjects) }\end{array}$ & $\begin{array}{c}N=6 \\
(48 \text { subjects })\end{array}$ & $\begin{array}{c}N=9 \\
(36 \text { subjects })\end{array}$ & $\begin{array}{c}N=12 \\
\text { (48 subjects) }\end{array}$ \\
\hline Session Averages & $0.20,0.18,0.25$ & $0.21,0.21,0.24$ & $0.13,0.20,0.15$ & 0.13 & 0.11 \\
Overall Average & 0.21 & 0.22 & 0.16 & 0.13 & 0.11 \\
Nash Prediction & 0.06 & 0.13 & 0.19 & 0.21 & 0.22 \\
\hline
\end{tabular}

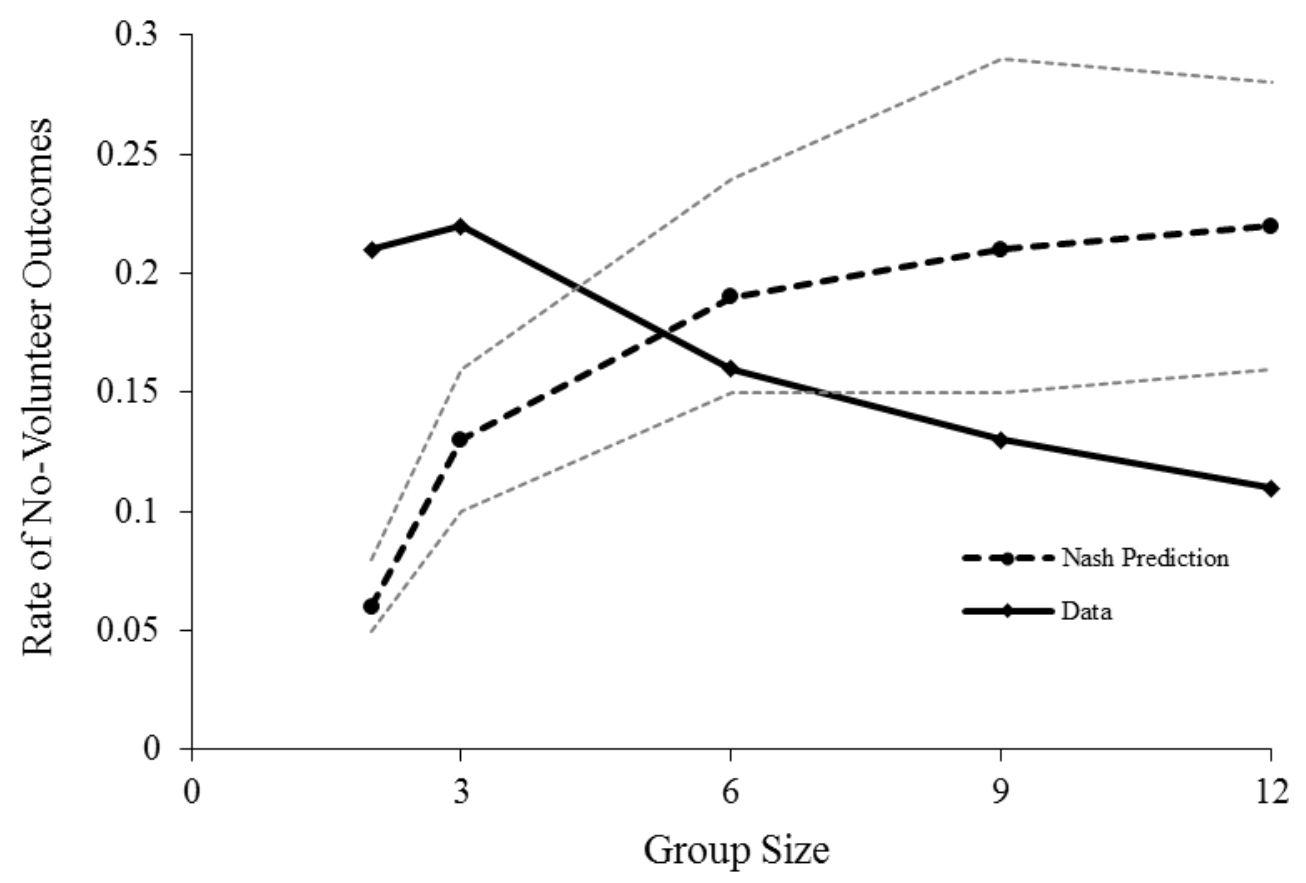

Figure 2. No-volunteer outcomes: Nash predictions (thick dashed line) with corresponding $95 \%$ confidence interval bounds (thin dashed lines) and data (solid line).

\footnotetext{
${ }^{7}$ These bounds are calculated as the rate of no-volunteer outcomes $(1-p)^{N}$ for the lower and upper bound volunteer probabilities reported in Figure 1.
} 
Note that the probability of a no-volunteer outcome does not generally increase with group size, as predicted by the Nash equilibria. In contrast, this probability is decreasing for the larger group sizes, as shown in Figure 2. These observations motivate our first result:

Result 1. Volunteer rates are declining in group size, as predicted in the symmetric Nash equilibrium. Volunteer rates, however, exhibit some "flatness" relative to predicted group size effects, and therefore, the rate of no-volunteer outcomes does not increase with group size as predicted.

Support. The null hypothesis of no group-size effect can be rejected at the 1 percent level, even if attention is restricted to session averages and qualitative rankings. ${ }^{8}$

One intuitive explanation for the declining rate of no-volunteer outcomes in the data is that there is some residual, non-strategic randomness in individual decisions that exceeds what is predicted in a symmetric Nash equilibrium. With very large groups, it is easy to imagine that someone will almost surely volunteer, whether it is due to distraction, error, or individual-specific factors. If this residual randomness puts a lower bound on the probability of volunteering, then the probability of no-volunteer outcomes will converge to 0 for very large groups. Residual non-strategic randomness would also tend to increase the variances of individual volunteer rates for all treatments. There is, of course, some variance even in a symmetric Nash equilibrium. In particular, if the Nash volunteer probability is $p$, then the predicted variances over 20 trials would be calculated as $20 p(1-p)$, which range from a variance of 5 for group size 3 to a variance of about 2 for group size 12. In contrast, the individual variances observed in the data are an order of magnitude higher (ranging between variances of about 45 for $N=2$ to about 19 for the larger groups. These variance numbers will be discussed in more detail in the next

\footnotetext{
8 There are 11 ! ways that the 11 session averages could be ranked. Under the null hypothesis of no group size effect, each of these 11 ! rankings is equally likely. The alternative hypothesis is that volunteer rates are lower for larger groups. This alternative makes no prediction of rankings within a treatment. For example, there are 6 ways that the three $N=2$ sessions can be ranked in the top group, and 6 ways that the three $N=3$ sessions can be ranked in the second group, and 6 ways that the three $N=6$ sessions can be ranked in the third group. This implies that there are $6 \times 6 \times 6=216$ ways that the session averages could be arrayed in a manner that is at least as extreme (in the direction of the alternative hypothesis) as what was observed. Since all 11! rankings would be equally likely under the null, the chances of seeing the ranking implied by the top row of Table 1 would be $216 /(11$ !), or about $0.005 \%$.
} 
section, where we consider equilibrium models of noisy behavior, and in section VI where we consider the equilibrium effects of unobserved heterogeneity.

\section{Noisy Behavior}

A standard way to model noisy behavior for simple binary choice decisions is to specify a probabilistic choice function (logit, probit, etc.) that maps expected payoff differences into choice probabilities. In an analysis of a game like the volunteer's dilemma, the expected payoffs themselves depend on beliefs about others' choices. The quantal response equilibrium (QRE) condition requires that the beliefs be rational in the sense that belief probabilities must correspond to choice probabilities (McKelvey and Palfrey, 1995). All players volunteer with probability $p$ in a symmetric equilibrium, and the resulting expected payoffs for volunteering and not volunteering are $\pi_{v}=V-C$ and $\pi_{n}=V\left(1-(1-p)^{N-1}\right)+L(1-p)^{N-1}$ respectively. For estimation purposes, we use a logit function:

$$
p=\frac{\exp \left(\lambda \pi_{v}\right)}{\exp \left(\lambda \pi_{v}\right)+\exp \left(\lambda \pi_{n}\right)},
$$

where $\lambda$ is a precision parameter. Recall that $\pi_{n}$ is a function of $p$ and $N$, so (5) is a nonlinear equation that must be solved numerically for $p$ as a function of $\lambda$ and $N$. We will denote the solution by $p(\lambda, N)$. The intuition is that the probability, $p$, that enters the expected payoff functions can be thought of as a "belief" and the $p$ on the left side of the logit formula in (5) is a choice probability. The equilibrium requires that these belief and choice probabilities be the same.

As $\lambda$ goes to zero, the right side of (5) converges to $1 / 2$ regardless of payoff differences, which corresponds to the case of perfectly noisy behavior. As $\lambda$ goes to infinity, it can be shown that the probability of making the best choice goes to 1 and thus the quantal response equilibrium volunteer probability converges to the symmetric Nash equilibrium. Recall that expected payoffs for each decision are equal in a mixed-strategy Nash equilibrium, and if $\pi_{v}=\pi_{n}$, the response determined by (5) is $p=1 / 2$. Thus if the Nash equilibrium involves probabilities of $1 / 2$ (as is the case for $N=3$ ), the quantal response prediction will also be $1 / 2$, regardless of the magnitude of $\lambda$. Moreover, when the Nash equilibrium is above or below $1 / 2$, the effect of adding noise via a quantal response 
will pull the prediction towards the midpoint of $1 / 2$ in these $n$-person binary-choice games. ${ }^{9}$ This "pull" to the center is somewhat reflected in the data plot for Figure 1.

To estimate the model, we specify the likelihood as a function of the QRE solution and the observed numbers of volunteer and no-volunteer decisions for each value of $N$. The data counts for volunteer decisions are denoted by $v(N)$ for group sizes $N=2$, $3,6,9$, and 12. The corresponding data counts for no-volunteer decisions are $n v(N)$. The likelihood function has a binomial structure, with the probabilities for each decision raised to a power that is the number of times that decision was observed. In particular, the likelihood is a constant (that we ignore in the maximization) times binomial expressions for each treatment:

$$
\Pi_{N=2,3,6,9,12} p(\lambda, N)^{v(N)}(1-p(\lambda, N))^{n v(N)}
$$

Then the log likelihood would be a constant plus a sum of decision counts times the logs of the associated QRE probabilities. At each iteration of the search process, the model must be solved for the quantal response probabilities, $p(\lambda, N)$ before evaluating the likelihood function at each stage of the search for the maximum value. The results are shown in the QRE Predictions row of Table 3.

Table 3. Maximum Likelihood Estimates for the QRE Precision (Standard Error)

\begin{tabular}{ccccc}
\hline \hline & \multirow{2}{*}{$\begin{array}{c}\text { Log } \\
\text { Precision }\end{array}$} & \multicolumn{2}{c}{ Predictions and Data Avg. $(N=2,3,6,9,12)$} \\
\cline { 4 - 5 } & & & Volunteer Rates & Rates of No-Volunteer Outcomes \\
\cline { 4 - 5 } & & & $.75, .50, .24, .16, .12$ & $.06, .13, .19, .21, .22$ \\
Symmetric Nash & & $.67, .50, .31, .24, .20$ & $.11, .13, .12, .08, .07$ \\
QRE & $11.0(.87)$ & -2392.4 & $.07, .40, .28, .19, .19$ & $.21, .22, .16, .13, .11$ \\
\hline
\end{tabular}

An alternative way to specify the model is in terms of an error parameter, $\mu$, which is the reciprocal of the precision. This error parameter estimate is 0.01 (the reciprocal of the precision estimate of 11), with a standard error of 0.01 , which is

\footnotetext{
${ }^{9}$ These results do not depend on the particular logit parametric specification. See Goeree and Holt (2005), who consider a more general case where the shocks to expected payoffs are independently and identically distributed. More precisely, they assume that the difference between the shocks has mean 0 and this difference has a cumulative distribution function with the property that $F(0)=1 / 2$.
} 
significantly greater than zero at the $1 \%$ level. The symmetric Nash equilibrium is a special case of QRE with infinite precision $(\mu=0)$, which supports the second result:

Result 2. The quantal response equilibrium provides a better fit to the data than the symmetric Nash equilibrium; it tracks some of the flatness in volunteer rates as group sizes increase, and it is qualitatively consistent with the tendency for no-volunteer outcomes to decline with large groups.

Recall that the symmetric Nash equilibrium volunteer probabilities decreased too sharply compared to the data averages, and that the equilibrium rates of no-volunteer outcomes increased with group size in direct contradiction to the data. The most interesting departures of the QRE predictions from symmetric Nash predictions are 1) the probabilities of no-volunteer outcomes on the right side of Table 3 are generally decreasing in group size, and 2) the extreme volunteer rates for groups sizes of 2 and 12 are pulled toward the center. Both of these departures tend to improve theoretical predictions. It is immediately apparent, however, that the predicted volunteer rates for QRE in the center row of the table (which range from 0.67 to 0.20 ) are too high for all group sizes, as compared with the data averages in the bottom row (which range from 0.52 to 0.19 ).

The symmetric Nash and QRE predictions differ even more sharply from the data in terms of variability. Recall that the variance of a binomial variable over $n$ trials with probability $p$ is: $n p(1-p)$. For example, if the predicted probability is 0.5 , as in both the QRE and symmetric Nash equilibria with group size 3, then the variance over 20 decision trials would be $20 / 4=5$, so the observed volunteer rates for a sample of 36 subjects in this treatments should be fairly tightly distributed around the predicted mean of 0.5 . The actual observed variance in individual volunteer rates for the 3-person treatment is 26.3, as shown in the bottom row of Table 4, and a similar difference is observed in the other treatments, with lower variance for larger group sizes. There are, of course, asymmetric Nash equilibria for the 3-person game, for example if one of the three people volunteers with probability 1 and the others with probability 0 , or if two people volunteer with probability 0.75 (as if it were a two-person group) and the other never volunteers. There would be no obvious way in which subjects in a random matching protocol could 
coordinate or even agree on these asymmetric patterns. Moreover, the implied variance of volunteer rates across people for these two asymmetric Nash equilibrium are about 91 (when only one person volunteers) or 54 (when only two people are volunteering with positive probability), both of which are much larger than the variance of volunteer rates, 26.3, that was observed for this treatment. Asymmetric equilibria will be discussed in more detail in the next section.

Table 4. Predicted and Observed Variances

Across Individual Volunteer Rates for 20 Trials

\begin{tabular}{cccccc}
\hline \hline & $\begin{array}{c}\text { Group size 2 } \\
\text { (34 subjects) }\end{array}$ & $\begin{array}{c}\text { Group size 3 } \\
\text { (36 subjects) }\end{array}$ & $\begin{array}{c}\text { Group size 6 } \\
(48 \text { subjects) }\end{array}$ & $\begin{array}{c}\text { Group size 9 } \\
\text { (36 subjects) }\end{array}$ & $\begin{array}{c}\text { Group size 12 } \\
(48 \text { subjects) }\end{array}$ \\
\hline Symmetric Nash Variance & 3.75 & 5.0 & 3.6 & 2.7 & 2.1 \\
\hline QRE Variance & 4.4 & 5.0 & 4.3 & 3.6 & 3.2 \\
\hline Observed Variance & 44.6 & 23.6 & 18.7 & 19.2 & 18.4 \\
\hline
\end{tabular}

An examination of the volunteer rates by subject reveals that this high variability is due to the fact that many subjects almost never volunteer, with rates close to zero, and others volunteer at a significant rate, although the observed volunteer rates for the non-freeriders are not clustered around the observed averages. The cumulative distributions of observed volunteer rates for each treatment are shown in the left panel of Figure 3, where the darker and gray lines are for the larger group sizes of 9 and 12. The analogous cumulative distributions calculated from QRE predictions are shown on the right side, where it is obvious that QRE predictions are not tracking heterogeneity in volunteer rates. 

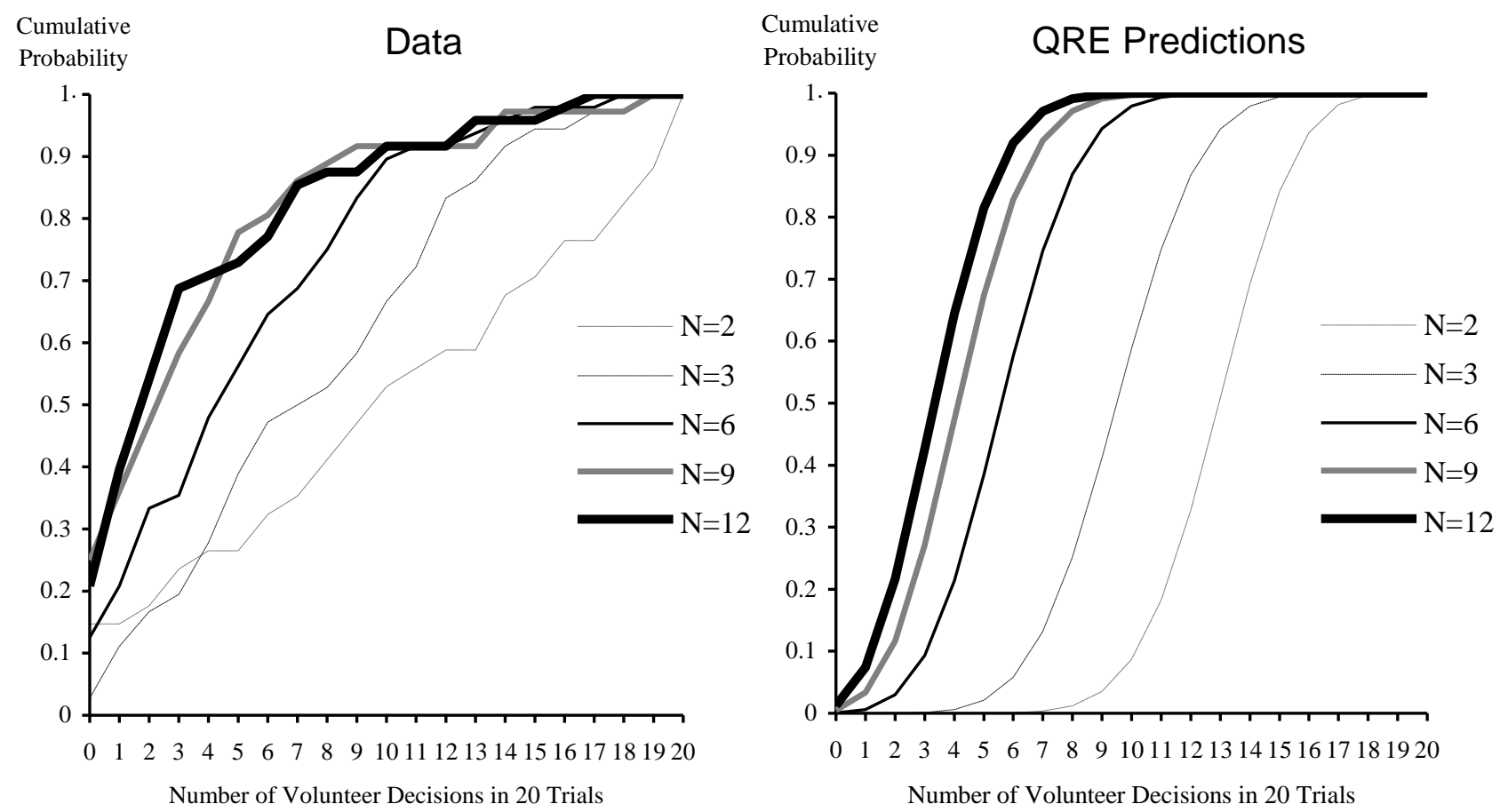

Figure 3. Cumulative Distributions of Volunteer Rates by Treatment:

Data in Left Panel and QRE Predictions in Right Panel

The cumulative distributions in the figure rise sharply on the left side of Figure 3 (near rates of 0 ), a feature that is most pronounced for large group sizes, which is consistent with the lower variances in Table 4 for larger groups. For example, the percentage of subjects that never volunteer ranges from $3 \%(N=3)$ to $25 \%(N=9)$. However, the majority of other individuals appear to be randomizing between volunteering and not. ${ }^{10}$ It is not uncommon for experimental papers that report conformity between theoretical predictions and data averages to mention "considerable heterogeneity" and behavioral differences that could be operating, but the patterns of variability differences in Table 4 and Figure 3 clearly call for more analysis.

\footnotetext{
${ }^{10}$ The percentages of subjects that either always volunteer or never volunteer are $26 \%, 3 \%, 13 \%, 25 \%$, and $21 \%$ for group sizes of $2,3,6,9$, and 12 , respectively. Of those that volunteer at least once, a runs test is unable to reject a random sequence of decisions for $88 \%, 97 \%, 90 \%, 96 \%$, and $95 \%$ of subjects in group sizes of $2,3,6,9$, and 12 , respectively. Thus, there does appear to be randomness in the sequence of individual decisions even though the incentive to be unpredictable is sharply diminished with random matchings as long as average probabilities are maintained, because subjects are unaware of the history of decisions of individuals with whom they are matched.
} 


\section{Individual Heterogeneity and QRE}

There are several potential explanations for the individual heterogeneity observed in the data. One possibility is that individuals vary in their propensity to volunteer or the individual psychological benefit they receive from the act of volunteering itself. This is analogous to a "warm glow" received from volunteering that can differ across individuals. In this case, the expected payoff to individual $i$ from volunteering can be modeled as $V-C+\delta_{i}$ where $\delta_{i}$ represents the warm glow propensity to volunteer, or cool glow propensity to free ride by not volunteering.

An alternative model of heterogeneity is that individuals differ in their aversion to volunteering unnecessarily, i.e. when another individual also chooses to volunteer. This captures the regret that might be felt when volunteering if there are other duplicate volunteers. ${ }^{11}$ This model of regret or "duplicate aversion" would imply that the expected payoff to individual $i$ from volunteering is $V-C-\gamma_{i}\left(1-(1-p)^{N-1}\right)$ where $\gamma_{i}$ represents the regret individual $i$ feels from volunteering when another person also volunteers, which occurs with probability $1-(1-p)^{N-1}$ assuming $p$ is the average volunteer probability across all others. Heterogeneous models incorporating both propensities to volunteer and regret will be explored in the context of a quantal response equilibrium. ${ }^{12}$

\footnotetext{
${ }^{11}$ This regret would not be surprising given that we estimate (using probit models) a significant negative effect of the fraction of group members an individual observes in the previous round on their volunteer choice in most treatments. This effect is stronger and more significant in treatments with larger group sizes (i.e. $N=9$ and $N=12$ ).

${ }^{12}$ The heterogeneous QRE models explored in this paper allow the heterogeneity to be modeled and interpreted as differences across individuals in their warm/cool glow propensity to volunteer or regret from unnecessarily duplicating the act of volunteering. Other heterogeneous QRE models instead allow for heterogeneity in the precision parameter $(\lambda)$ across individuals (i.e. Rogers, Palfrey, and Camerer, 2009). Another possibility is based on the idea that people have heterogeneous beliefs. It is our view that people do not form precise but different beliefs about equilibria, but rather, they learn from experience based on their own encounters with prior random groupings. In this manner, different people will have different observed "histories." So histories determine beliefs via some learning model, and beliefs determine quantal responses, which in turn, determine how histories evolve. The steady state choice distributions for this process is a "stochastic learning equilibrium" as applied in Capra et al (2002) and analyzed more fully in Goeree, Holt, and Palfrey (2016, chapter 5), who prove existence using an assumption that players have finite memories. For example, an extreme special case would be Cournot best responses, which involve 1period memories. For the price-competition game considered in Capra et al. (2002), the stochastic learning equilibrium distribution is a little flatter than the quantal response equilibrium for the game, due to the extra bit of randomness that arises from different individual histories. An alternative approach to belief heterogeneity is the Friedman and Mezzetti (2005) random belief equilibrium in which each person chooses a best response to their own belief profile that is a random draw from a distribution of possible random belief profiles.
} 
Specifically, following Goeree, Holt, and Laury (2002), we model individual heterogeneity as a discrete truncated normal distribution. The individual propensities $\left(\delta_{1}\right)$ and regrets $\left(\gamma_{\mathrm{i}}\right)$ are calculated on a range around a mean $\mu$ (plus or minus one standard deviation $\sigma$ ). This range is divided into 11 points: $\mu-\sigma, \mu-.8 \sigma, \mu-.6 \sigma, \ldots \mu, \ldots \mu+\sigma$. The normal density is evaluated at each of these points, and each density value is divided by the sum of all values, which yields a discrete "bell-shaped" distribution on this interval. The resulting densities will be denoted by $f(t, \mu, \sigma)$ for $t=1, \ldots 11$. The approach is to use the probabilities of the 11 individual "types" to evaluate a weighted average of the quantal response probabilities for each type. In equilibrium, this average response must equal the volunteer probability that was used to compute expected payoffs for each decision. For each group-size treatment $N(=2,3,6,9,12)$ and for each parameter set $\lambda, \mu, \sigma$, the QRE solution $p(\lambda, \mu, \sigma, N)$ is calculated by minimizing the sum of squared "belief errors" (distances between the belief probabilities and the associated weighted average quantal responses). Then these QRE solutions (with belief errors that are essentially zero) are used to evaluate the likelihood function as the program searches for the parameter set that maximizes the likelihood. ${ }^{13}$

The final aspect of the estimation that differs from the previously considered homogeneous case is the structure of the likelihood function. With heterogeneity, we use data counts for each individual, which will be denoted by $v(i, N)$ and $n v(i, N)$ for the numbers of volunteer and no-volunteer decisions for person $i$ in treatment $N(=2,3,6,9$, 12). Thus the data counts are both person-specific and treatment-specific. The likelihood is a product of binomials, analogous to (6), using the response probabilities that depend on all parameters, the treatment, and the individual type $t$. These type-specific response probabilities will be denoted by $p(\lambda, \mu, \sigma, N, t)$. The products of the binomials are taken over all treatments and all $n(N)$ subjects in each treatment with group size $N$. The terms in the square brackets in (7) are sums of binomials for each type $t$ of each person, weighted by the type probability $f(t, \mu, \sigma)$. The likelihood is a product (across treatments and people) of type-weighted sums for each person.

\footnotetext{
${ }^{13}$ Goeree, Holt, and Laury (2002) previously estimated a quantal response model of voluntary contributions to a public good with a truncated normal distribution of individual's altruism parameters. In that case, however, the linear structure of the payoffs in the game made it unnecessary to solve the model at each stage of the estimation, since terms involving beliefs about others' contributions canceled out of the relevant quantal response formulas.
} 


$$
\Pi_{N=2,3,6,9,12} \prod_{i=1 . n(N)}\left[\Sigma_{t=1 \ldots 11} p(\lambda, \mu, \sigma, N)^{v(i, N)}(1-p(\lambda, \mu, \sigma, N, t))^{n v(i, N)} f(t, \mu, \sigma)\right] .
$$

The estimation results are shown in Table 5, where the top QRE row shows the estimates obtained previously for the simple model with no heterogeneity. The next two rows show estimates for two models with heterogeneity, the propensities model and the regret model. The maximized log likelihoods are much larger than for the simple model, and the predicted volunteer rates and no-volunteer outcomes on the right side of the table are much closer to the data averages at the bottom.

Table 5. Maximum Likelihood Estimates (Standard Errors)

Note the negative mean propensity to volunteer of -0.20 in the second row of the table, which indicates that most people are averse to volunteering. This aversion is analogous to "warm glow" altruism (but in the opposite direction) in that it is modeled to be independent of the structural parameters. The HQRE Regret model, in contrast, has an

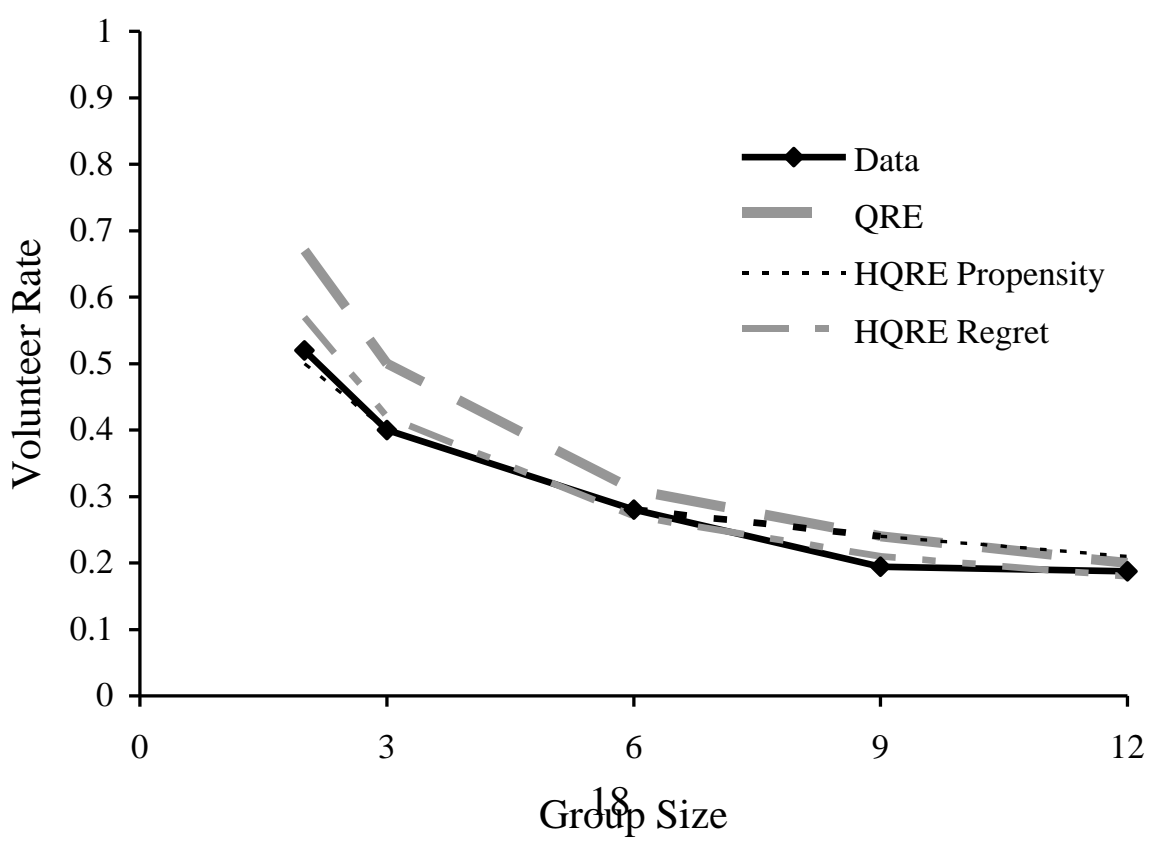


aversion that depends on the structural feature associated with the probability of a duplicate volunteer decision. The predictive accuracies for these two models in terms of likelihood and of the predicted volunteer and no-volunteer rates are quite good, as shown in Figures 4 and 5. Both heterogeneous models track the data more closely than the base QRE model, especially for low group sizes. However, it appears that the HQRE Regret predictions are closer to the observed data in most cases, both in Figure 4 where the "dash-dot" line virtually overlaps the dark data line for volunteer rates, and in figure 5 where the dash-dot no-volunteer rate has the same shape as the data line.

Figure 4. Volunteer Probabilities: QRE and HQRE predictions (both with volunteer propensities and regret) and observed data averages

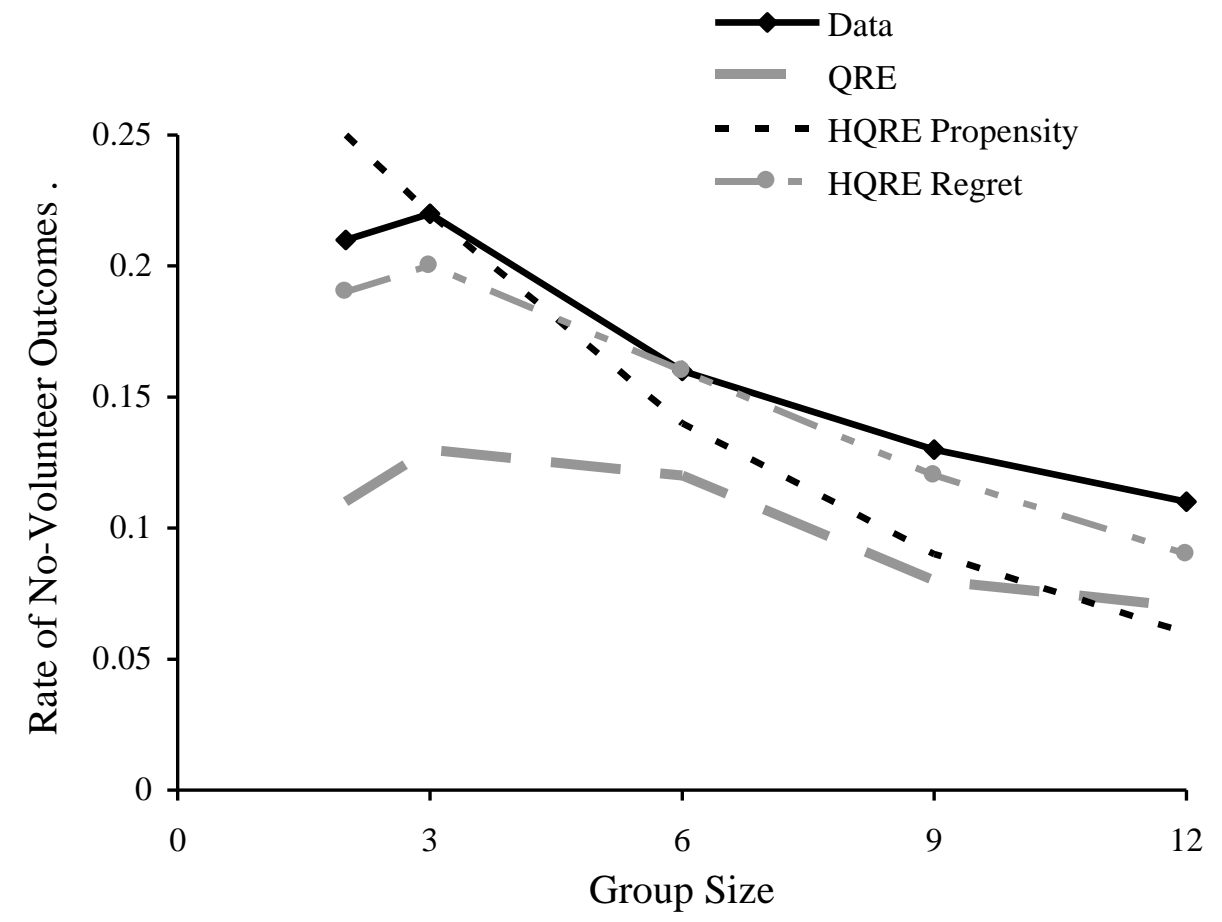

Figure 5. No-Volunteer Rates: QRE and HQRE predictions (both with volunteer propensities and regret) and observed data averages

Finally, the predicted cumulative distributions of volunteer rates for the two models are shown in Figure 6, with Volunteer Propensities on the left and Regret 
(duplicate aversion) on the right. These distributions exhibit some of the salient features of the data distributions shown previously in Figure 3. In particular, the vertical intercepts in both panels of Figure 6 imply a large proportion of individuals who never volunteer, which is especially pronounced for larger group sizes (9 and 12) as represented by the darker lines. In both panels, there is not much difference between the distributions for the two larger group sizes, but the distributions for the smaller group sizes (2 and 3) are well separated and closer to uniformity (a 45 degree line). These observations are roughly consistent with the cumulative data distributions on the left side of Figure 3. The main feature of the data distributions in Figure 3 that is not well predicted is the slight tendency for extreme decisions (always or never volunteer) in groups of size 2 that is seen in the short dashed-line cumulative on the left side of Figure 3.
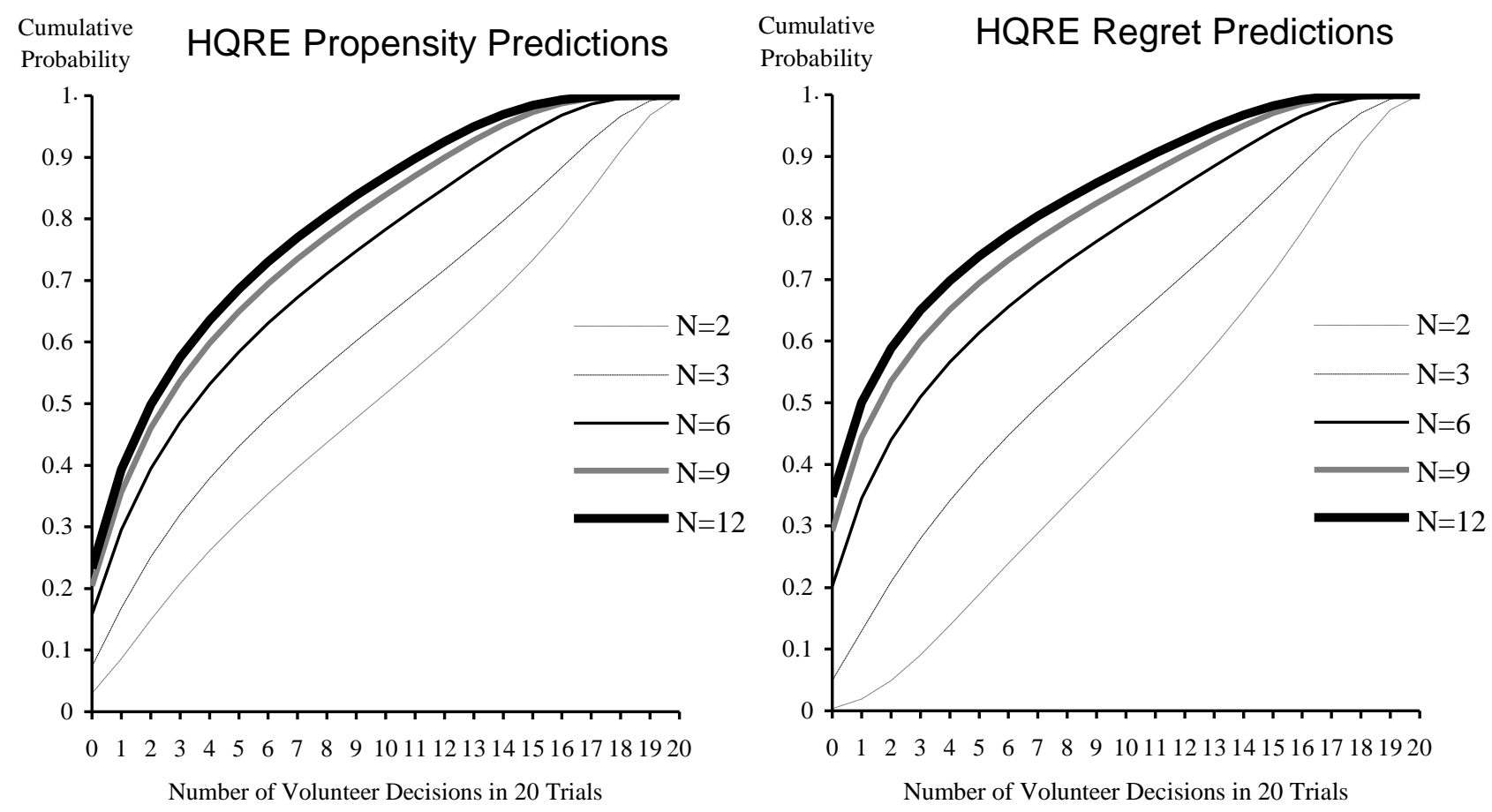

Figure 6. Cumulative Distributions of Predicted Volunteer Rates for Heterogeneous QRE by Treatment: Propensities (Left Panel) and with Regret (Right Panel)

The parameter estimates for the HQRE models can be used to generate predicted variances in individual volunteer rates, and these variances are in the same overall range 
$(20 \%-38 \%)$ as the observed variances of $45,24,19,19,18$ for group sizes of 2, 3, 6, 9, and 12 respectively. ${ }^{14}$ These observations can be summarized:

Result 3. The estimated heterogeneous QRE models track both the data averages (volunteer rates and rates of no-volunteer outcomes) closely. Both HQRE models imply variances in individual volunteer rates that are in the approximate range of observed variances and that compare favorably with simple QRE variances that are an order of magnitude too low.

Support: The visual fits for volunteer rates and no-volunteer outcomes provided in Figures 4 and 5 are consistent with a dramatic improvement on the log likelihood shown in Table 5, which lowers the Akaike information criterion (calculated as 2 times the number of estimated parameters minus 2 times the log likelihood) from 4786.8 (QRE) to 3974.4 (HQRE-regret) and 3951.4 (HQRE-propensities). ${ }^{15}$

As noted earlier, there are asymmetric Nash equilibria in which some people randomize and others never volunteer, which would also result in heterogeneity. The online appendix reports results of Komogorov-Smirnoff tests of whether the observed distribution of volunteer rates is different from the theoretical distribution predicted by the various Nash equilibria. The Kolmogorov-Smirnov test results indicate that the majority of (even asymmetric) Nash equilibria produce volunteer rate distributions that are significantly different from the data. Those that are not significantly different often produced very different variances than what is observed. The most notable exception is when 5 people mix when $N=9$. It would be difficult for subjects to coordinate on specific asymmetric equilibria in the presence of randomly changing group compositions. Also, while it is apparent from Figure 3 that there are a number of individuals who never volunteer (especially for $N=9$ and $N=12$ ), the density at a volunteer rate of 0 is not nearly large enough to correspond to what is predicted by many of the asymmetric equilibria in the appendix. Our view is that there are many possible degrees of

\footnotetext{
${ }^{14}$ For the HQRE "propensities" model, the predicted variances are 38, 36, 27, 23, and 20 for groups of size $2,3,6,9$, and 12. The corresponding variance predictions are 29, 34, 28, 24, 20 for the HQRE "regret" model. In these heterogeneous models (HQRE Regret and HQRE Propensity), there is a probability distribution over types that volunteer with different probabilities. Thus, to compute the predicted variance, we simulated the types and associated volunteer decisions (depending on estimates of $\lambda, \mu$, and $\sigma$ ) for the same number of subjects that took part in each treatment of the experiment. In total, 1000 groups were simulated for each treatment and these represent the average variances over those 1000 simulations.

${ }^{15}$ All of these Akaike information criteria are lower than the AIC associated with the symmetric Nash equilibria (4923.6).
} 
asymmetry with some people volunteering more than others, and the HQRE model provides an endogenous determination of the degree of variability that permits out-ofsample predictions, to be discussed next.

\section{An Out-of-Sample Test}

As a robustness check, we ran two additional treatments to evaluate the various predictions out of sample. The procedures were the same as those described in the procedures section, except that the volunteer cost was cut in half, from $\$ 0.20$ to $\$ 0.10$, in one case and the volunteer cost was doubled, from $\$ 0.20$ to $\$ 0.40$, in a second treatment. Each treatment involved 15 subjects, randomly matched for 20 rounds with group size 3. Table 6 shows the data and predictions for Nash, QRE, and HQRE, based on the previously estimated parameter values ( $\lambda$ and type distribution mean and standard deviation, $\mu$ and $\sigma$ ) taken from Table $5 .{ }^{16}$

Table 6. Out-of-Sample Data and Theoretical Predictions

\begin{tabular}{|c|c|c|c|c|c|c|}
\hline & & $\begin{array}{c}\text { Symmetric } \\
\text { Nash }\end{array}$ & QRE & $\begin{array}{c}\text { HQRE } \\
\text { Propensity }\end{array}$ & $\begin{array}{l}\text { HQRE } \\
\text { Regret }\end{array}$ & $\begin{array}{c}\text { Data } \\
\text { (15 subjects) }\end{array}$ \\
\hline & Average Volunteer Rate & 0.65 & 0.59 & 0.45 & 0.48 & 0.49 \\
\hline \multirow[t]{3}{*}{$\begin{array}{c}\text { Low Cost } \\
\text { Treatment } \\
\text { C }=0.10\end{array}$} & $\begin{array}{c}\text { Rate of No-Volunteer } \\
\text { Outcomes }\end{array}$ & 0.04 & 0.07 & 0.17 & 0.14 & 0.15 \\
\hline & $\begin{array}{c}\text { Variance of Volunteer } \\
\text { Rates }\end{array}$ & 4.55 & 4.84 & 37.78 & 38.28 & 32.35 \\
\hline & Average Volunteer Rate & 0.29 & 0.35 & 0.31 & 0.31 & 0.29 \\
\hline \multirow[t]{2}{*}{$\begin{array}{l}\text { High Cost } \\
\text { Treatment } \\
\text { C=0.40 }\end{array}$} & $\begin{array}{l}\text { Rate of No-Volunteer } \\
\text { Outcomes }\end{array}$ & 0.36 & 0.27 & 0.33 & 0.33 & 0.35 \\
\hline & $\begin{array}{c}\text { Variance of Volunteer } \\
\text { Rates }\end{array}$ & 4.12 & 4.55 & 29.69 & 20.82 & 20.17 \\
\hline
\end{tabular}

${ }^{16}$ Recall that the variance for symmetric Nash and QRE are computed using the formula for the variance of a binomial variable over 20 trials: $n p(1-p)$. This is because all individuals in this case are assumed to volunteer with the same probability. As discussed in more detail in footnote 14, the variances for the HQRE models reported in this table are also computed using simulations (1000 simulations of 15 subjects each). 
The two heterogeneous models continue to predict well out-of-sample. Notice that, in the low-cost treatment, the symmetric Nash (0.65) and QRE (0.59) predictions of average volunteer rates are much higher than the predictions of the HQRE models $(0.45$ and 0.48 ) but that the data average is much closer to the HQRE predictions. On the contrary, in the high-cost treatment, the symmetric Nash (0.29) and QRE (0.35) predicted average volunteer rates are very similar to the predictions of the HQRE models $(0.31)$ and the observed volunteer rate $(0.29)$ is relatively close to all of these theoretical predictions. In addition, the variances associated with both the symmetric Nash equilibrium and QRE are much smaller than the observed variance in individual volunteer rates (out of 20 trials) for both treatments. The observed variances are much closer to the predictions provided by the HQRE models.

\section{Conclusion}

The key strategic feature of the volunteer's dilemma game is that all players, however many there are, enjoy the benefit if at least one of them incurs the cost of providing the public good. The game is motivated by applications where a single person's decision can be decisive, e.g. casting a politically costly veto, attempting a dangerous rescue, or sounding an alarm that increases one's risk of being attacked by a predator. This situation also describes some standard psychology experiments with "staged emergencies," where it is observed that volunteer rates decline in large groups, a pattern that is attributed to "diffusion of responsibility." Game theory provides a formal model based on a similar idea; the expected benefit of volunteering (holding the volunteer rate constant) is lower in a large group of potential volunteers, causing equilibrium volunteer rates to be decreasing in group size. In fact, the predicted probability of volunteering declines so sharply that the probability of getting no volunteers at all is greater for large groups.

This unintuitive prediction is tested in the context of a laboratory experiment in which subjects are arrayed into groups (with sizes of 2,3,6,9, and 12) in a process of random matchings over 20 rounds of play. The data patterns show that volunteer rates decline as the group size increases, as predicted, but that this decline is not as sharp as predicted in the symmetric Nash equilibrium, and hence, that the probability of getting no volunteers at all is generally decreasing, not increasing, in group size. The intuition is 
that some residual non-strategic randomness may pull volunteer rates up when they are very low, which reduces the chance of no-volunteer outcomes for large group sizes. A stochastic (quantal response) equilibrium is estimated for the data; this model is promising, but it overestimates volunteer rates for low group sizes and does not explain high variability across subjects (it is off by an order of magnitude). Much of the observed heterogeneity in the data is due to differences in individuals' propensities or aversions to volunteering. A heterogeneous QRE with a two-parameter distribution of individual propensities or "types" fits the data patterns well, in terms of both the overall averages and the variability around the averages. Finally, the improved performance of the QRE models with heterogeneity holds up well in an out-of-sample test done with 3person groups by doubling the volunteer cost or cutting the cost in half. 


\section{References}

Babcock, L., M.P. Recalde, L. Vesterlund, and L. Weingart (2016) “Gender Differences in Accepting and Receiving Requests for Tasks with Low Promotability," forthcoming in the American Economic Review.

Bergstrom, Ted, Rod Garratt, and Greg Leo (2015) "Motivations of Competing Altruists," Discussion Paper, UCSB.

Capra, C.M, J.K. Goeree, R. Gomez, C.A. Holt (2002): "Learning and Noisy Equilibrium Behavior in an Experimental Study of Imperfect Price Competition," International Economic Review, 43(3), 613-636.

Darley, J.M. and Latane, B. (1968) "Bystander Intervention in Emergencies: Diffusion of Responsibility," Journal of Personality and Social Psychology, 8, 377-383.

Diekmann, A. (1985) "Volunteer's Dilemma," Journal of Conflict Resolution, 29, 605610.

Diekmann, A. (1986): "Volunteer's Dilemma: A Social Trap without a Dominant Strategy and Some Empirical Results," in Paradoxical Effects of Social Behavior: Essays in Honor of Anatol Rapoport, ed. by A. Diekmann, and P. Mitter. Heidelberg: Physica-Verlag, 187-197.

Diekmann, A. (1993): "Cooperation in Asymmetric Volunteer's Dilemma Game: Theory and Experimental Evidence," International Journal of Game Theory, 22, 75-85.

Diekmann, A., and P. Mitter (1986): Paradoxical Effects of Social Behavior: Essays in Honor of Anatol Rapoport, Heidelberg and Vienna: Physica-Verlag, 341.

Franzen, A. (1995): “Group Size and One Shot Collective Action,” Rationality and Society, 7, 183-200.

Friedman, James W. and Claudio Mezzetti (2005) "Random Belief Equilibrium in Normal Form Games," Games and Economic Behavior, 51, 296-323.

Goeree, J.K. and C.A. Holt (2005): "An Explanation of Anomalous Behavior in Models on Political Participation," American Political Science Review, 99(2), 201-213.

Goeree, J.K., C.A. Holt, and S.K. Laury (2002): "Private Costs and Public Benefits: Unraveling the Effects of Altruism and Noisy Behavior," Journal of Public Economics, 83(2), 257-278. 
Goeree, J.K., C.A. Holt, and T.R. Palfrey (2003): "Risk Averse Behavior in Asymmetric Matching Pennies Games," Games and Economic Behavior, 45, 97-114.

Goeree, J.K., C.A. Holt, and T.R. Palfrey (2016): Quantal Response Equilibrium: A Stochastic Theory of Games, Princeton NJ: Princeton University Press.

Healy, A. and J. Pate (2009): "Asymmetry and Incomplete Information in an Experimental Volunteer's Dilemma," In Anderssen, R.S., R.D. Braddock and L.T.H. Newham (eds) 18th World IMACS Congress and MODSIM09 International Congress on Modelling and Simulation, pp. 1459-1462. ISBN: 9780-9758400-7-8.

Latane, B. and J.M. Darley (1970): The Unresponsive Bystander: Why Doesn't He Help? New York: Appleton-Century-Crofts.

McKelvey, R.D. and T.R. Palfrey (1995): "Quantal Response Equilibrium for NormalForm Games," Games and Economic Behavior, 10, 6-38.

Murnigham, J.K., J.W. Kim, and A.R. Metzger (1993): "The Volunteer's Dilemma," Administrative Science Quarterly, 38(4), 515-538.

Otsubo, H. and A. Rapoport (2008): Dynamic Volunteer's Dilemmas over a Finite Horizon - An Experimental Study," Journal of Conflict Resolution, 52(6), 961984.

Rogers, Brian W. and Palfrey, Thomas R. and Camerer, Colin F (2009): "Heterogeneous Quantal Response Equilibrium and Cognitive Hierarchies," Journal of Economic Theory, 144 (4), 1440-1467.

Siegel, Sidney and N. John Castellan, Jr. (1988): Nonparametric Statistics for the Behavioral Sciences, $2^{\text {nd }}$ edition, New York: McGraw-Hill. 


\section{Instructions Appendix}

- Rounds and Matchings: The experiment sets up 3-person groups which interact in a sequence of rounds. Note: In each round, you will be matched with 2 people selected at random from the other participants. There will be new random groupings each round.

- Interdependence: The decisions that you and the 2 other people make will determine your earnings.

- Decisions: Both you and the other 2 people will decide whether to incur a cost to undertake an action that may benefit all of you. You cannot see the others' decisions while choosing yours, and vice versa.

- Duplication: The full benefit from this action is available to all if at least one person undertakes the costly action, and no additional benefit is accrued if more than one person incurs this cost.

- Player Costs: The cost of the action will be the same for all people in each group.

\section{Continue}

- Investment Cost: In each period or "round", you will decide whether to make a costly decision, which we will refer to as an investment. If you decide to invest, you incur a cost of $\mathbf{\$ 0 . 2 0}$ in the current round. If your decision is not invest, you incur no cost.

- Investment Benefit: If at least one person in your group decides to invest, all people in the group will receive an amount, $\mathbf{\$ 1 . 0 0}$, whether or not they invested in that round.

- Earnings: If at least one person invests, then a person who invests earns $\mathbf{\$ 1 . 0 0}-\mathbf{\$ 0 . 2 0}$, and a person who does not invest earns $\mathbf{\$ 1 . 0 0}$. If nobody invests, they all earn $\mathbf{\$ 0 . 2 0}$.

- Initial Capital: All of you start the first round with an earnings balance of $\mathbf{\$ 0 . 0 0}$. Earnings in each round will be added to this amount. The program will keep track of cumulative earnings for all rounds.

\section{Continue}

In the following examples, please use the mouse button to select the best answer. Remember that the cost of investing is $\mathbf{\$ 0 . 2 0}$, and the benefit if at least one person invests is $\mathbf{\$ 1 . 0 0}$. If none of the 3 people in your group invest, all earn $\mathbf{\$ 0 . 2 0}$.

Question 1: Suppose at least one person invests and the others in your group do not. Then:

a) a person who invests earns $\mathbf{\$ 0 . 8 0}$ and the others earn $\mathbf{\$ 1 . 0 0}$

b) a person who invests earns $\$ \mathbf{1 . 0 0}$ and the others earn $\$ \mathbf{0 . 8 0}$ 
Question 2: If all of you invest, you each earn (X); if none of you invest, you each earn (Y).

a) $X=\$ 1.00, Y=\$ 0.20$

b) $X=\$ 0.80, Y=\$ 0.20$

\section{Continue}

Question 1: Suppose at least one person invests and the others in your group do not. Then:

(6)

(a) a person who invests earns $\$ 0.80$ and the others earn $\$ 1.00$

C

(b) a person who invests earns $\mathbf{\$ 1 . 0 0}$ and the others earn $\mathbf{\$ 0 . 8 0}$

Your answer, (a) is Correct. A person who invests earns $\mathbf{\$ 1 . 0 0}-\mathbf{\$ 0 . 2 0}=\mathbf{\$ 0 . 8 0}$, and a person who does not invest earns $\$ \mathbf{1 . 0 0}$.

Question 2: If all of you invest, you each earn (X); if none of you invest, you each earn (Y).

(a) $X=\$ 1.00, Y=\$ 0.20$

(b) $\mathrm{X}=\$ 0.80, \mathrm{Y}=\$ 0.20$

Your answer, (b) is Correct since the cost $\mathbf{\$ 0 . 2 0}$ must be deducted from the payoff $\mathbf{\$ 1 . 0 0}$ when one invests.

\section{Summary of Instructions}

- Matchings: At the beginning of each round, there will be a new random grouping of all participants, so the 2 people who you are matched with in one round may not be the same people you will be matched with in the subsequent round. Matchings are random, and you are no more likely to be matched with some people than with others.

- Earnings: All people will begin a round by choosing an investment decision, which entails a cost of $\mathbf{\$ 0 . 2 0}$. If at least one person in your group chooses to invest, all will receive a payment of $\mathbf{\$ 1 . 0 0}$, and your earnings will be this payment minus your investment cost (if any). If nobody invests, all receive $\mathbf{\$ 0 . 2 0}$.

- Cumulative Earnings: Everybody begins with an initial cash balance of $\mathbf{\$ 0 . 0 0}$. Earnings will be added to this amount. The program will keep track of your cumulative earnings.

- Rounds: There will be a number of rounds, with random rematchings at the beginning of each. 
Online Appendix: Data and Predictions for Symmetric and Asymmetric Nash Equilibria

\begin{tabular}{|c|c|c|c|c|c|c|c|c|}
\hline $\begin{array}{l}\text { Group } \\
\text { Size } \\
\mathrm{N}\end{array}$ & $\begin{array}{c}\text { Number of } \\
\text { Individuals } \\
\text { Volunteering } \\
\text { with Positive } \\
\text { Probability }\end{array}$ & $\begin{array}{l}\text { Volunteer Rate } \\
\text { (for those } \\
\text { volunteering } \\
\text { with positive } \\
\text { probability) }\end{array}$ & $\begin{array}{c}\text { Expected } \\
\text { Average } \\
\text { Volunteer } \\
\text { Rate }\end{array}$ & $\begin{array}{c}\text { Observed } \\
\text { Volunteer } \\
\text { Rate }\end{array}$ & $\begin{array}{c}\text { Expected } \\
\text { Variance } \\
\text { (Simulated for } \\
\text { Asymmetric } \\
\text { Equilibria) }\end{array}$ & $\begin{array}{l}\text { Observed } \\
\text { Variance }\end{array}$ & $\begin{array}{c}\text { Kolmogorov- } \\
\text { Smirnoff Test } \\
\text { Statistic on } \\
\text { Individual } \\
\text { Volunteer Rates }\end{array}$ & $\begin{array}{l}\text { Significance } \\
\quad * * * 1 \% \\
\quad * * 5 \% \\
* 10 \%\end{array}$ \\
\hline \multirow[t]{2}{*}{2} & 1 & 1.00 & 0.50 & \multirow[t]{2}{*}{0.52} & 103.0 & \multirow[t]{2}{*}{44.6} & $\mathrm{D}=.3824$ & $* * *$ \\
\hline & 2 & 0.75 & 0.75 & & 3.8 & & $\mathrm{D}=.5179$ & $* * *$ \\
\hline \multirow[t]{3}{*}{3} & 1 & 1.00 & 0.33 & \multirow[t]{3}{*}{0.40} & 91.4 & \multirow[t]{3}{*}{23.6} & $\mathrm{D}=.6389$ & $* * *$ \\
\hline & 2 & 0.75 & 0.50 & & 54.1 & & $\mathrm{D}=.4321$ & $* * *$ \\
\hline & 3 & 0.50 & 0.50 & & 5.0 & & $\mathrm{D}=.4146$ & $* * *$ \\
\hline \multirow[t]{6}{*}{6} & 1 & 1.00 & 0.17 & \multirow[t]{6}{*}{0.28} & 56.7 & \multirow[t]{6}{*}{18.7} & $\mathrm{D}=.7083$ & $* * *$ \\
\hline & 2 & 0.75 & 0.25 & & 52.0 & & $\mathrm{D}=.5417$ & $* * *$ \\
\hline & 3 & 0.50 & 0.25 & & 27.8 & & $\mathrm{D}=.3750$ & $* * *$ \\
\hline & 4 & 0.37 & 0.25 & & 15.6 & & $\mathrm{D}=.2084$ & $* *$ \\
\hline & 5 & 0.29 & 0.24 & & 8.1 & & $\mathrm{D}=.1710$ & \\
\hline & 6 & 0.24 & 0.24 & & 3.7 & & $\mathrm{D}=.2290$ & $* *$ \\
\hline \multirow[t]{9}{*}{9} & 1 & 1.00 & 0.11 & \multirow[t]{9}{*}{0.19} & 40.6 & \multirow[t]{9}{*}{19.2} & $\mathrm{D}=.6389$ & $* * *$ \\
\hline & 2 & 0.75 & 0.17 & & 40.4 & & $\mathrm{D}=.5278$ & $* * *$ \\
\hline & 3 & 0.50 & 0.17 & & 24.0 & & $\mathrm{D}=.4167$ & $* * *$ \\
\hline & 4 & 0.37 & 0.16 & & 16.0 & & $\mathrm{D}=.3056$ & $* * *$ \\
\hline & 5 & 0.29 & 0.16 & & 10.8 & & $\mathrm{D}=.1950$ & \\
\hline & 6 & 0.24 & 0.16 & & 7.9 & & $\mathrm{D}=.0898$ & \\
\hline & 7 & 0.21 & 0.16 & & 5.6 & & $\mathrm{D}=.1123$ & \\
\hline & 8 & 0.18 & 0.16 & & 3.9 & & $\mathrm{D}=.1595$ & \\
\hline & 9 & 0.16 & 0.16 & & 2.7 & & $\mathrm{D}=.2194$ & $*$ \\
\hline \multirow[t]{12}{*}{12} & 1 & 1.00 & 0.08 & \multirow[t]{12}{*}{0.19} & 31.2 & \multirow[t]{12}{*}{18.4} & $\mathrm{D}=.7083$ & $* * *$ \\
\hline & 2 & 0.75 & 0.13 & & 32.7 & & $\mathrm{D}=.6250$ & $* * *$ \\
\hline & 3 & 0.50 & 0.13 & & 20.5 & & $\mathrm{D}=.5417$ & $* * *$ \\
\hline & 4 & 0.37 & 0.12 & & 14.0 & & $\mathrm{D}=.4584$ & $* * *$ \\
\hline & 5 & 0.29 & 0.12 & & 10.2 & & $\mathrm{D}=.3754$ & $* * *$ \\
\hline & 6 & 0.24 & 0.12 & & 7.6 & & $\mathrm{D}=.2932$ & $* * *$ \\
\hline & 7 & 0.21 & 0.12 & & 6.4 & & $\mathrm{D}=.2136$ & $* *$ \\
\hline & 8 & 0.18 & 0.12 & & 4.8 & & $\mathrm{D}=.1934$ & $*$ \\
\hline & 9 & 0.16 & 0.12 & & 3.9 & & $\mathrm{D}=.2064$ & $* *$ \\
\hline & 10 & 0.14 & 0.12 & & 3.0 & & $\mathrm{D}=.2286$ & $* *$ \\
\hline & 11 & 0.13 & 0.12 & & 2.6 & & $\mathrm{D}=.2369$ & $* * *$ \\
\hline & 12 & 0.12 & 0.12 & & 2.1 & & $\mathrm{D}=.2448$ & $* * *$ \\
\hline
\end{tabular}

